\title{
Clinical and surgical-pathological staging in early non-small cell lung cancer
}

\author{
loannis Koukis, ${ }^{1}$ loannis Gkiozos, ${ }^{2}$ Ioannis Ntanos, ${ }^{2}$ Elias Kainis, ${ }^{2}$ Konstantinos N. Syrigos ${ }^{2}$ \\ ${ }^{1}$ Department Cardiothoracic Surgery, 401 Army General Hospital, Athens; ${ }^{2}$ Oncology Unit, GPP, \\ Medical School of Athens, Sotiria General Hospital, Athens, Greece
}

\begin{abstract}
Staging is of the utmost importance in the evaluation of a patient with non-small cell lung cancer (NSCLC) because it defines the actual extent of the disease. Accurate staging allows multidisciplinary oncology teams to plan the best surgical or medical treatment and to predict patient prognosis. Based on the recommendation of the International Association for the Study of Lung Cancer (IASLC), a tumor, node, and metastases (TNM) staging system is currently used for NSCLC. Clinical staging (c-TNM) is achieved via non-invasive modalities such as examination of case history, clinical assessment and radiological tests. Pathological staging (p-TNM) is based on histological examination of tissue specimens obtained with the aid of invasive techniques, either non-surgical or during the intervention. This review is a critical evaluation of the roles of current pre-operative staging modalities, both invasive and non-invasive. In particular, it focuses on new techniques and their role in providing accurate confirmation of patient TNM status. It also evaluates the surgical-pathological staging modalities used to obtain the true-pathological staging for NSCLC.
\end{abstract}

\section{Introduction}

Lung cancer is responsible for more cancer deaths than the next

Correspondence: Konstantinos N. Syrigos, Oncology Unit GPP, Sotiria General Hospital, Athens Medical School 152, Mesogeion Av., Athens, Greece.

E-mail: ksyrigos@med.uoa.gr

Key words: clinical staging, pathological staging, non-small cell lung cancer.

Contributions: the authors contributed equally.

Conflict of interests: the authors declare no potential conflict of interests.

Received for publication: 31 March 2013.

Revision received: 14 July 2013.

Accepted for publication: 26 August 2013.

This work is licensed under a Creative Commons Attribution NonCommercial 3.0 License (CC BY-NC 3.0).

CC Copyright I. Koukis et al., 2013

Licensee PAGEPress, Italy

Oncology Reviews 2013; 7:e7

doi:10.4081/oncol.2013.e7 three most common cancers combined. Each year, $29 \%$ and $24 \%$ of cancer deaths in males and females, respectively, are attributed to lung cancer. It also represents $15 \%$ and $14 \%$, in males and females, respectively, of all new cases of cancer. ${ }^{1}$ In particular, non-small cell lung cancer (NSCLC) accounts for approximately 75-85\% of all newly diagnosed lung cancers. ${ }^{2}$ Surgery remains the best chance for a cure if an early diagnosis is made. However, only one-third of patients are operable at the time of diagnosis. Accurate staging is required to provide precise information about the extent of the disease and to determine the most appropriate therapy in patients with NSCLC. ${ }^{3}$ It is also important for estimating prognosis and for clinical trials. The TNM staging system, together with guidelines from the European and North American societies and study groups, is currently used and is usually expressed as clinical and pathological staging.

Clinical staging is carried out before surgery and several different modalities are adopted. It is a truly multidisciplinary process involving imaging, medical and surgical techniques. It determines whether the patient has an early stage tumor and may proceed to direct resection. ${ }^{4}$ The important weapons in the armamentarium of the multidisciplinary oncology team include: computed tomography (CT), magnetic resonance imaging (MRI), mediastinoscopy, transthoracic, transbronchial or transesophageal fine needle aspiration (FNA) with or without ultrasound guidance, core needle biopsy, and positron emission tomography (PET) together with CT (PET-CT). The multidisciplinary oncology team employs a variety of specialists, including thoracic surgeons, oncologists and respiratory specialists. The objective remains the most accurate assessment of the stage of NSCLC in order to choose the best treatment approach. The increased use of new techniques should reduce errors in classification of clinical staging when compared against the available gold standard of pathological staging.

Surgical-pathological staging represents the results of histology for all the specimens obtained during surgical resection of NSCLC. It involves the tumor, and hilar and mediastinal lymph nodes from different sites obtained either by taking samples or by complete excision. These specimens can be stained with conventional methods or with immunohistochemical indices and examined under the microscopy. The result represents the true stage of the NSCLC and is used to plan further treatment.

This review critically evaluates the roles of current pre-operative staging modalities, both invasive and non-invasive. It also evaluates the surgical-pathological staging modalities to obtain the true-pathological staging for NSCLC.

\section{Clinical staging of non-small cell lung cancer}

In NSCLC, the anatomical extent of disease, as described by the TNM classification, is essential as it predicts prognosis, dictates treatment modalities, and provides a standardized description of the dis- 
ease which facilitates research and the comparison of outcomes across clinical trials. ${ }^{5}$ TNM staging has evolved since its inception in 1974 when 2155 cases from a single center were used to devise the staging system. Today, data from 67,725 cases from 46 sources in various countries have been pooled and validated by the International Association for the Study of Lung Cancer (IASLC) in a massive undertaking to reclassify NSCLC based on survivorship. The result is a more discriminatory classification system than that used in years past. The seventh edition of the TNM classification was published at the end of 2009, and came into effect from $1^{\text {st }}$ January 2010 (Tables 1-4). ${ }^{6}$

Although there have been significant changes in some $\mathrm{T}$ and $\mathrm{M}$ descriptors (Table 5) and the resultant TNM stage groupings, the most significant change in this revision has been the process of revision itself. ${ }^{7-12}$ Accurate staging involves imaging and tissue acquisition, both of which require a working knowledge of the attributes of these diagnostic modalities.

\section{Non-invasive clinical staging}

All NSCLC patients should have a thorough case history taken and undergo a complete physical examination that, together with a routine array of laboratory tests (such as alkaline phosphatase), constitute the expanded clinical evaluation. ${ }^{13}$ Abnormal findings from such an evaluation are associated with an approximate $50 \%$ incidence of abnormal findings on subsequent imaging. ${ }^{13}$ In the absence of abnormal findings on clinical evaluation, there is little evidence to support the use of routine imaging to detect extra thoracic metastases. ${ }^{14}$ Nevertheless, further investigation is usually required to provide sufficient data for accurate TNM staging. The ideal staging investigation should be inexpensive and easy to administer, have high sensitivity and specificity, provide accurate results that reflect the patient's true clinical condition, and yet cause minimal discomfort and morbidity. ${ }^{15,16}$ Hence noninvasive staging by imaging, the least invasive approach, typically makes up the first line of investigation. It is currently recognized that chest radiographs are not sufficiently sensitive or reliable in this role. ${ }^{17}$ The latest guidelines from the American College of Chest Physicians (ACCP) recommend that further assessment (i.e. chest CT) be performed in virtually all NSCLC patients except those for whom extreme debilitation precludes further evaluation or treatment. ${ }^{18}$

\section{Chest radiograph}

Most cases of lung cancers are initially detected on a plain chest radiograph. In some situations, the plain radiograph may be sufficient to detect spread of the tumor to the mediastinum. For example, the presence of bulky lymphadenopathy in the superior or contralateral mediastinal areas may be considered adequate evidence of metastatic disease, precluding a further imaging evaluation of the chest. This may be particularly true if the patient is too ill or is unwilling to undergo treatment of any kind. However, it is recommended that tissue confirmation be

Table 1. Tumor descriptors (modified from Sobin et al., 20106).

Tx Primary tumor cannot be assessed, or tumor proven by the presence of malignant cells in sputum or bronchial washings, but not visualized by imaging or bronchoscopy

T0 No evidence of primary tumor

T1 Tumor $\leq 3 \mathrm{~cm}$ (max. dimension) surrounded by lung or visceral pleura, without bronchoscopic evidence of invasion more proximal than the lobar bronchus (i.e. not in the main bronchus)

Tla Tumor $<2 \mathrm{~cm}$ (max. dimension). The uncommon superficial spreading tumor of any size with its invasive component limited to the bronchial wall, which may extend proximal to the main bronchus, is also classified as Tla

Tlb Tumor $>2 \mathrm{~cm}$ but not $>3 \mathrm{~cm}$ at its max. dimension

T2 Tumor $>3 \mathrm{~cm}$ but not $>7 \mathrm{~cm}$ (T2 tumors with these features are classified T2a if $\leq 5 \mathrm{~cm}$ or if size cannot be determined, and T2b if $>5 \mathrm{~cm}$ but not $>7 \mathrm{~cm}$ ); or tumor with any of the following features: i) involves main bronchus, $2 \mathrm{~cm}$ or more distal to the carina; ii) invades visceral pleura; iii) associated with atelectasis or obstructive pneumonitis that extends to the hilar region but does not involve the entire lung

T3 Tumor $>7 \mathrm{~cm}$ or that directly invades any of the following: chest wall (including superior sulcus tumors), diaphragm, phrenic nerve, mediastinal pleura, parietal pericardium; or tumor in the main bronchus $<2 \mathrm{~cm}$ distal to the carina but without involvement of the carina; or associated atelectasis or obstructive pneumonitis of the entire lung or separate tumor nodule(s) in the same lobe as the primary

T4 Tumor of any size that invades any of the following: mediastinum, heart, great vessels, trachea, recurrent laryngeal nerve, esophagus, vertebral body, carina; separate tumor nodule(s) in a different ipsilateral lobe to that of the primary

Table 2. Lymph node descriptors (modified from Sobin et al., 20106).

\begin{tabular}{ll}
\hline NX & Regional lymph nodes cannot be assessed \\
N0 & No regional lymph node metastasis \\
\hline N1 & Metastasis in ipsilateral peribronchial and/or ipsilateral hilar lymph nodes and intrapulmonary nodes, including involvement by direct extension \\
N2 & Metastasis in ipsilateral mediastinal and/or subcarinal lymph node(s) \\
\hline N3 & Metastasis in contralateral mediastinal, contralateral hilar, ipsilateral or contralateral scalene, or supraclavicular lymph node(s) \\
\hline
\end{tabular}

Table 3. Metastatic descriptors (modified from Sobin et al., 20106).

\begin{tabular}{ll}
\hline M0 & No distant metastasis \\
M1 & Distant metastasis \\
\hline Mla & Separate tumor nodule(s) in a contralateral lobe; tumor with pleural nodules or malignant pleural or pericardial effusion \\
\hline
\end{tabular}


obtained when possible by the least invasive method available. It is widely accepted that the chest radiograph is, in general, an insensitive measurement of mediastinal lymph node involvement with lung cancer; thus, further non-invasive and/or invasive assessment is usually necessary. ${ }^{18}$

\section{Thorax computed tomography}

Chest CT is often the first step in staging NSCLC because of the detailed anatomical information obtained. A CT scan can define the location, size, and anatomical characteristics of a tumor much better than a chest radiograph can. CT is useful for delineating the local extent and invasion of a lung tumor, e.g. into the chest wall or mediastinum. The long axis of the primary lesion is measured. If surgical treatment is not appropriate, the long axis of the tumor is subsequently followed according to the Response Evaluation Criteria in Solid Tumors (RECIST). Three-dimensional reconstructions can be created from CT scan data, providing a clearer picture of tumor morphology. Standard CT protocols, including the upper liver and adrenal glands, are also said to detect metastatic deposits in these organs in $3-10 \%$ of asymptomatic patients. Intravenous contrast enhancement is desirable as it helps to distinguish lymph nodes from vascular structures. ${ }^{19}$ A normal-sized mediastinal node is defined as a lymph node with a short axis diameter of $1 \mathrm{~cm}$ or under on a transverse CT scan image. Various CT criteria have been suggested to define features suggestive of malignant involvement, but none including the size criteria are very sensitive or specific. Almost $20 \%$ of the lymph nodes smaller than $1 \mathrm{~cm}$ are malignant and nearly $40 \%$ of lymph nodes larger than $1 \mathrm{~cm}$ are benign. ${ }^{18-20}$ In one meta-analysis, the pooled sensitivity of chest CT for detecting mediastinal lymph node metastatic involvement using size criteria is only 51\% [95\% confidence interval (CI): $47-54 \%$ ] and pooled specificity is $86 \%$ (95\% CI: $84-88 \%$ ) in a population with a median prevalence of $28 \%{ }^{18}$ Interestingly, the specificity of CT scans can be influenced by confounding factors that lead to anatomic changes, such as post obstructive pneumonia. ${ }^{21}$ In spite of the low accuracy of CT scan in diagnosing mediastinal nodal metastasis, the importance of anatomical information obtained from a CT scan cannot be under-emphasized as it helps with further decision making and in identifying the appropriate biopsy site. Most current guidelines recommend a CT scan in the initial evaluation of NSCLC.

\section{Magnetic resonance imaging}

Magnetic resonance imaging is of limited additional value when CT is used in the evaluation of a primary lung tumor or mediastinal lymph nodes. Occasionally, chest MRI may be helpful in determining the presence of mediastinal, chest wall, vertebral, or vascular invasion.

The role of MRI in the metastatic workup for lung cancer is a subject of debate. Head MRI appears to be more sensitive than CT in detecting brain metastases. ${ }^{13} \mathrm{~T} 1$ - and T2-weighted MRI images may be helpful in distinguishing between benign and malignant liver and adrenal lesions. In cases in which PET-CT scan is needed for staging of NSCLC, brain MRI is an adequate substitute.

\section{Positron emission tomography-computed tomography scan}

Whole PET scan provides functional imaging by evaluating the preferential uptake of radiolabeled 18fluorodeoxyglucose (18F-FDG) by tumor cells and combined PET-CT improves this technology further by adding the anatomical details to the functional image. A standard uptake value cut off of over 2.5 is considered abnormal and suggestive of neoplastic involvement. This applies mostly when there is suspicion of mediastinal lymph nodes..22 A large number of studies evaluating accuracy of staging methods and already six meta-analyses $s^{6,14-18}$ have demonstrated that PET is superior to CT scans for mediastinal staging in potentially operable NSCLC. ${ }^{14,23,24}$ PET has been shown to refine lung cancer staging and prevent futile thoracotomies in as many as $20 \%$ of patients who were otherwise considered candidates for surgery. ${ }^{25}$ Significant improvements in tumor staging were found with integrated PET-CT scan. The anatomical correlation of the radionuclide

Table 4. Stage groupings for non-small cell lung cancer (modified from Sobin et al., 20106).

\begin{tabular}{ll}
\hline Occult carcinoma & TX - N0 - M0 \\
Stage 0 & Tis - N0 - M0 \\
\hline Stage IA & Tla,b - N0 - M0 \\
Stage IB & T2a - N0 - M0 \\
\hline Stage IIA & T2b - N0 - M0; Tla, b - N1 - M0; T2a - N1 - M0 \\
Stage IIB & T2b - N1 - M0; T3 - N0 - M0 \\
\hline Stage IIIA & Tla,b, T2a,b - N2 - M0; T3 - N1, N2 - M0; T4 - N0, N1 - M0 \\
Stage IIIB & T4 - N2 - M0; any T - N3 - M0 \\
\hline Stage IV & Any T - any N - M1 \\
\hline
\end{tabular}

Table 5. Changes to the tumor, node and metastasis (TNM) descriptors in the seventh edition of the TNM classification of non-small cell lung cancer (modified from ${ }^{7-12}$ ).

A new 2-cm cutoff now divides T1 tumors into Tla $<2 \mathrm{~cm}$ and T1b tumors $>2$ $\mathrm{cm}$ but not $>3 \mathrm{~cm}$

A new cutoff of $5 \mathrm{~cm}$ divides T2 tumors into T2a $>3 \mathrm{~cm}$ but not $>5 \mathrm{~cm}$ and T2b tumors $>5 \mathrm{~cm}$ but not $>7 \mathrm{~cm}$

A new 7-cm cutoff was created, and tumors $>7 \mathrm{~cm}$ are classified as T3, size becoming a T3 descriptor for the first time

Tumors associated with additional tumor nodules in the same lobe as the primary are reclassified from $\mathrm{T} 4$ to $\mathrm{T} 3$

Tumors associated with additional tumor nodules in other ipsilateral lobe(s) are reclassified from M1 to T4

Tumors associated with additional tumor nodules in the contralateral lung remain Ml, but are reclassified as Mla

Tumors associated with malignant pleural or pericardial effusion or pleural or pericardial nodules are reclassified from T4 to Mla

Tumors associated with distant metastases are reclassified as Mlb

Table 6. Invasive modalities used for lymph node mapping according to the International Association for the Study of Lung Cancer (IASLC) staging committee (modified from Vansteenkiste et al., 2010 ${ }^{4}$ ).

\begin{tabular}{|c|c|}
\hline $\begin{array}{l}\text { Invasive lymph node } \\
\text { assessment modalities }\end{array}$ & $\begin{array}{l}\text { Lymph node stations that can be } \\
\text { assessed }\end{array}$ \\
\hline EUS-FNA & 2R, 2L, 4L, 5, 7, 8, 9, 10R, 10L \\
\hline EBUS-TBNA & 2R, 2L, 4R, 4L, 7, 10R, 10L, 11R/11L \\
\hline Cervical mediastinoscopy & 2R, 2L, 4R, 4L, 7, 10R \\
\hline Anterior mediastinotomy & 5,6 \\
\hline Left-sided VATS & $5,6,8,9$ \\
\hline
\end{tabular}


uptake made it possible to obtain a more precise delineation of the location of the primary tumor. Integrated PET-CT improved the diagnosis of chest-wall infiltration and mediastinal invasion. However, despite the fact that integrated PET-CT improved the accuracy of mediastinal staging, the resolution of PET is not sufficient to detect microscopic lymph-node metastases. If the radionuclide uptake is not increased on PET, integrated PET-CT will certainly not provide further information. ${ }^{26}$ A recent randomized Canadian trial of 337 patients by Maziak and colleagues ${ }^{27}$ comparing PET-CT with conventional staging (CT abdomen and bone scan) in suspected early stage NSCLC on routine CT (all patients also had brain CT or MRI) demonstrated that PET-CT correctly up-staged disease in $13.8 \%$ of patients as compared with only $6.8 \%$ in the conventional staging group $(\mathrm{P}=0.046)$ and prevented unnecessary thoracotomies. National Comprehensive Cancer Network (NCCN) evidenced-based clinical practice guidelines suggest that PET-CT scan should be considered in patients with clinical stage IA NSCLC being treated with curative intent, to evaluate for mediastinal and extra thoracic staging. Patients with clinical IB-IIIB lung cancer being treated with curative intent should also undergo PET-CT scan for mediastinal and extra thoracic staging. However, it is clearly stated that in patients with an abnormal result on FDG-PET scans, further evaluation of the mediastinum with sampling of the abnormal lymph node should be performed prior to surgical resection of the primary tumor.

Some important considerations regarding PET-CT need to be made. False-negative PET findings must be managed with extra care in some particular situations, i.e. little FDG avidity of the primary tumor, presence of a central tumor or of centrally located N1 nodes, both of which may obscure nearby existing mediastinal lymph node infiltration. False-positive findings have also been reported. This is due to the fact that FDG uptake is not tumor specific, and can be found in all active tissues with high glucose metabolism, in particular inflammation. The efficacy of mediastinal lymph node staging via PET-CT has been thoroughly studied. Positive prognosis rates are $47-100 \%$ while the negative prognostic value is $86-95 \%{ }^{28}$ Therefore, clinically relevant FDGavid mediastinal lymph nodes should always be examined with the most appropriate tissue sampling technique. ${ }^{4}$

\section{Invasive clinical staging of non-small cell lung cancer}

\section{Endoscopy}

Standard white-light bronchoscopy (WLB) is considered mandatory in patients with suspected lung cancer. In addition to pathological confirmation in many patients, it also permits an evaluation of the endobronchial extension of the tumor to be made (endobronchial T stage) which can be decisive in determining the extent of resection or for planning radiotherapy. ${ }^{4}$ WLB is usually accompanied by washing, brushing and direct biopsy of the intrabronchial lesion in an attempt to obtain further molecular information about the tumor. Autofluorescence bronchoscopy (AFB) added to WLB has a role in the routine workup of patients suspected of having operable lung cancer based on chest imaging or in patients with newly diagnosed lung cancer planned for resection. ${ }^{29}$ Adding AFB to WLB may reveal synchronous multi-centricity of pre-invasive and radio-occult invasive lesions in $10 \%$ of the patients in whom a primary radiographically visible invasive lung cancer was detected. Nowadays, video-AFB systems are available, facilitating access to anatomic and functional information at the time of the first bronchoscopy without significantly increasing examination time. ${ }^{4}$

Another useful modality for the pre-operative staging of NSCLC is the conventional or blind transbronchial needle aspiration (TBNA). For the $\mathrm{N}$ stage, blind TBNA can be performed during the initial standard bronchoscopy if enlarged LNs are present on CT. A blind TBNA is most often applied to selected LN levels, i.e. those with clear anatomical landmarks, such as lower paratracheal LNs in position 4, subcarinal LNs in position 7, and hilar LNs in position 11 right and left. When they are clearly enlarged (at least $15 \mathrm{~mm}$ short axis diameter), they can be adequately aspirated using a needle through the working channel of a standard bronchoscope. ${ }^{30}$ As such, a blind TBNA could be useful in clinical stage II to establish the diagnosis of lung cancer based on the TBNA of an enlarged hilar lymph node, but there is no reason to perform a blind TBNA for pre-operative mediastinal LN staging in early stage I/II NSCLC. TBNA can be combined with a new technique of direct histological diagnosis called rapid on site evaluation that is performed during endoscopy. The benefit is that it is easier to obtain a sample for biopsy (in $50 \%$ of cases only one attempt is required). It also has better diagnostic accuracy than classic TBNA and a sensitivity of $97 \%{ }^{31,32}$

The advent of endoscopic ultrasonography has allowed imaging beyond the mucosa into the mediastinum, e.g. visualization of LNs in the vicinity of the esophagus, trachea or main bronchi, and has, therefore, improved the accuracy of endoscopic mediastinal LN sampling techniques. Endobronchial ultrasonography (EBUS) can be performed with a linear echo-endoscope under local anesthesia using moderate sedation. It is able to visualize superior and inferior mediastinal LNs at levels $2 \mathrm{R} / 2 \mathrm{~L}, 4 \mathrm{R} / 4 \mathrm{~L}$ and 7 , as well as hilar LNs at level 10,11 and even 12. EBUS helps to localize these LNs and perform a TBNA under realtime ultrasonographic control. The mediastinal LN stations accessible with EBUS-TBNA are the same as for cervical mediastinoscopy. Esophageal ultrasonography (EUS) uses an echo-endoscope with linear array ultrasound transducer at the tip, keeping the working channel of the endoscope available to pass a needle and perform a FNA under ultrasonographic control. This technique visualizes, in particular, superior mediastinal LNs in level $4 \mathrm{~L}$, and inferior mediastinal LNs in levels 7, 8 and 9, as described on the new LN map. ${ }^{33}$ This complements other techniques, as several of these LNs (levels 8 and 9) are not accessible by EBUS-TBNA or mediastinoscopy. Mediastinal lymph node station $4 \mathrm{R}$ is often a blind spot for EUS-FNA because of the interposition of the trachea. On the other hand, EUS-FNA can sample hilar LN station $10 \mathrm{R}$ or $10 \mathrm{~L}(\mathrm{N1})$, in which case one has to be extremely careful not to consider these LN stations as mediastinal (N2) LNs. However, the majority of the non-randomized trials studied the potential of echoendoscopic techniques for the mediastinal staging of clinical N2/3 lung cancer. Clinical data focusing on the value of endoscopic ultrasonography for the N0/1 NSCLC are scarce. A non-randomized study reported a high accuracy of EBUS-TBNA in patients with lung cancer and a normal mediastinum on CT and PET. ${ }^{34}$

For clinical implementation, an important issue is that EBUS-TBNA (like US-FNA) has a suboptimal negative predictive value ranging from $60 \%$ to $80 \%$, which requires a confirmatory surgical staging procedure in the case of a non-malignant echo-endoscopic needle aspiration. № false-positive mediastinal lymph node findings by EBUS-TBNA have been reported in literature, and all but one EUS-FNA series reported no false-positive needle aspirations. ${ }^{35}$ A false-positive finding can, however, occur in the case of: i) contamination of cytological material when the needle passes dysplastic mucosa (e.g. EBUS-TBNA through mucosa of viral bronchitis) or neoplastic mucosa (e.g. EUS-FNA through malignant esophageal mucosa); ii) misclassification of activated/enlarged lymphocytes as suspicious epithelial cells by the cytopathologist; or iii) sample of primary tumor tissue mistakenly taken instead of mediastinal LN material (e.g. in the case of a central hilar tumor adjacent to the mediastinal LN). It is clear that cervical mediastinoscopy remains the first choice base-line invasive mediastinal staging test in patients with clinical early stage I and II lung cancer requiring pre-operative invasive mediastinal staging. Additional clinical research is needed to establish the value of mediastinal staging by endoscopic ultrasonography in subsets of patients with early stage I and II lung cancer. 


\section{Thoracocentesis and transthoracic needle biopsy}

Thoracocentesis is a useful modality for all patients with suspected or diagnosed NSCLC who present with a pleural effusion. In most instances, it provides an easy and safe method for ruling out the presence of a malignant effusion. Unfortunately, only $50-65 \%$ of diagnostic thoracocentesis are conclusive for malignancy. In such cases, further evaluation with video-assisted thoracic surgery (VATS) or medical thoracoscopy may be needed.

Thoracocentesis and transthoracic needle biopsy (TTNB) of a suspicious pulmonary nodule is usually performed by interventional radiologists with the assistance of a cytopathologist. A positive yield as high as $95 \%$ has been reported by some experienced centers. Most centers, however, report an accuracy rate of $75-90 \%$. However, this modality is mandatory in the initial evaluation of a pulmonary nodule. If the result is negative but the other clinical findings present strong suspicion of malignancy, the pulmonary lesion can be further evaluated by means of PET-CT scan or VATS. TTNB is also useful in establishing the diagnosis for adrenal lesions found on CT or PET-CT scan.

\section{Cervical mediastinoscopy}

Cervical mediastinoscopy remains a key tool for staging the upper mediastinal LNs in patients with early stage I/II lung cancer. It is a surgical biopsy technique under general anesthesia. ${ }^{36}$ The mediastinoscope is inserted through a small suprasternal incision. Blunt dissection then gives access to the pretracheal, right and left paratracheal, and anterior subcarinal LNs. There was no internationally accepted recommendation on how many LN stations should be examined at cervical mediastinoscopy. The guidelines of the

European Society of Thoracic Surgeons (ESTS) now recommend systematic exploration and biopsy of the right and left paratracheal and the subcarinal LNs. In addition, if present, the upper paratracheal LNs should be sampled and biopsied. ${ }^{37}$ In experienced hands, the average sensitivity of cervical mediastinoscopy to detect mediastinal LN involvement is approximately $80 \%$ according to a recent review, with a high NPV of $89 \%{ }^{38}$ The results of the suboptimal sensitivity are partly explained by the fact that some $\mathrm{LN}$ stations $(5,6,7$ posterior, 8,9$)$ are not accessible by cervical mediastinoscopy. Other advantages of cervical mediastinoscopy are that it allows a complete mapping of mediastinal LNs, discrimination between extra- and intracapsular LN disease, and between nodal disease and direct invasion of the mediastinum by the tumor itself. More recently, the introduction of videomediastinoscopes has improved visualization, allowing findings to be recorded, and improving teaching possibilities. ${ }^{4}$

\section{Anterior mediastinotomy}

Left upper lobe tumors are known to metastasize predominantly to the aortopulmonary window and para-aortic LNs (levels 5 and 6). These LN stations cannot be reached by cervical mediastinoscopy, and need either left anterior mediastinotomy or left thoracoscopy (see below). The mediastinotomy procedure is more demanding and has a higher morbidity than the cervical approach. When a cervical mediastinoscopy is negative, this procedure may be indicated in cases of high suspicion of involvement of LN level 5 or 6 (e.g. in the case of enlarged or FDGavid LNs in that area). ${ }^{4}$

\section{Video-assisted thoracic surgery}

VATS surgical thoracoscopy) can be a useful addition to cervical mediastinoscopy, as it allows one to reach subcarinal nodes or inferior mediastinal nodes on the right side, and para-aortic nodes or inferior mediastinal nodes on the left side. For VATS, the false-negative rate was $15 \%$ both in enlarged and normal sized nodes with a wide variation in sensitivity of from $37 \%$ to $100 \%{ }^{38}$ The advantage over left anterior mediastinotomy is that anatomical landmarks such as the vagal and phrenic nerve are more easily recognized. There are no recent series on the use of VATS for staging of mediastinal nodes, which probably reflects the fact that less invasive staging methods such as EUS-FNA have become the preferred technique for staging of inferior mediastinal $\mathrm{LNs}^{4}$ (Table 6).

\section{Surgical-pathological staging}

\section{Intraoperative staging}

Although it is admitted that nodal staging of non-small cell lung cancer should be as accurate as possible, the extent of mediastinal lymph node assessment during surgery is controversial and there is no consensus. Different techniques are used, ranging from simple visual inspection of the unopened mediastinum to an extended bilateral lymph node dissection. Furthermore, different terms are used to define these techniques. ${ }^{39}$

The ESTS has issued guidelines for intraoperative lymph node staging in an attempt to organize and clarify this issue. It is now well accepted that a removal of at least six lymph nodes from hilar and mediastinal stations is recommended to define nodal staging accurately and to determine pN0 status. ${ }^{40}$ Furthermore, there is evidence that multilevel and multi-nodal disease or extra capsular involvement has a poorer prognosis. Thus, lymph node staging techniques can be categorized as follows:

- Sampling: the removal of one or more lymph nodes guided by pre- or intraoperative findings that are thought to be representative. Systematic sampling means a pre-determined selection of the lymph node stations specified by the surgeon. Sampling, either random or selective, is plagued by inconsistency. In random sampling, only the obviously enlarged mediastinal nodes are removed, i.e. the ones that can be clearly visualized or palpated. On the other hand, systematic sampling involves obtaining lymph node tissue from all or almost all mediastinal stations but not the entire lymph node tissue from each station. Thus, the amount and quality of specimens is highly dependent on the surgeon and cannot be accurately measured.

- Systematic nodal dissection: all the mediastinal tissue containing the lymph nodes is dissected and removed systematically within anatomical landmarks. It is recommended that at least 3 mediastinal nodal stations (but always subcarinal) should be excised as a minimum requirement. The nodes are separately labeled and examined histologically. Besides the mediastinal nodes, the hilar and the intrapulmonary lymph nodes are dissected as well. ${ }^{40}$

- Lobe-specific systematic node dissection: the mediastinal tissue containing specific lymph node stations are excised, depending on the lobar location of the primary tumor. The subcarinal front should always be removed.

- Extended lymph node dissection: bilateral mediastinal and cervical lymph node dissection is performed through median sternotomy and cervicotomy. ${ }^{23}$

For complete resection of non-small cell lung cancer, a systematic nodal dissection is recommended in all cases (also after induction therapy). ${ }^{41}$ On the right side, upper mediastinal node stations $2 \mathrm{R}$ and $4 \mathrm{R}$ should be removed along with the fatty tissue from the diaphragm to the subcarinal space, thus effectively removing stations 7,8 and 9 . On the left side, removal of the lymph node stations 4-9 should be performed, including the sub-aortic (aortopulmonary window, number 5) and para-aortic (number 6) stations. For a complete nodal dissection of the left upper mediastinum, division of the ligamentum arteriosus allowing mobilization of the aortic arch is recommended, with special 
care not to damage the left recurrent laryngeal nerve. ${ }^{39}$ Each of the nodal stations excised should be placed in separate vials with appropriate labeling for histological analysis. Here it is important to note that, despite the recommendations for systematic mediastinal lymph node dissection, possibly fewer than one-third of the thoracic surgeons practising worldwide perform this type of lymphadenectomy. For peripheral squamous T1, a more selective nodal dissection depending on the lobar location of the primary tumor (lobe-specific systematic nodal dissection) is acceptable, based on the detailed analysis of lobe-specific lymphatic drainage published by Naruke et $a .^{42}$ and Ichinose $e t a l .^{43}$ It has been shown that the probability of unforeseen N2 disease is very low $(<5 \%)$ in such patients. ${ }^{44}$ Therefore, for this specific type of early NSCLC, the following lobe-specific node dissection can be performed: i) right upper and middle lobe: $2 \mathrm{R}, 4 \mathrm{R}$ and 7 ; ii) right lower lobe: $4 \mathrm{R}, 7$, 8 and 9; iii) left upper lobe: 5, 6 and 7; iv) left lower lobe: 7,8 and 9 .

In total, the lymphadenectomy specimen should include at least six nodes.

After induction therapy, the same recommendation for lymph node assessment should be applied. However, lymph node dissection in the upper mediastinum after induction therapy (especially chemotherapy or radiotherapy after previous mediastinoscopy) may be technically difficult.

Intraoperative lymph node assessment can be minimized in highrisk patients undergoing minimally invasive video-assisted wedge resections, but if the patient can tolerate a lobectomy, standard recommendation of lymph node assessment should be followed. ${ }^{45}$ Whether extending the lymph-node dissection influences survival or recurrence rate of the disease remains to be determined. ${ }^{29}$ There are data which clearly show that systematic sampling or nodal dissection improves intraoperative staging, especially in the detection of multi-level N2 disease which is associated with a poorer prognosis. ${ }^{46,47}$

\section{Histopathological evaluation of the removed lymph nodes}

Recommendations about the histopathological evaluation of the lymph nodes were published in 2001 by the Association of Directors of Anatomic and Surgical Pathology. ${ }^{48}$ However, in practice, there is no established consensus. The analysis of the nodes depends on the center and on the pathologist, and it is often difficult to find a compromise between theoretical demands and practical feasibility. Some recommendations can be given to define quality criteria for this evaluation.

As a first step, all resected intrapulmonary, hilar, and mediastinal nodes should be examined macroscopically. In the presence of gross tumour, one hematoxylin-eosin stained section should be performed at the most macroscopically suspicious site to demonstrate the metastasis and its possible extra capsular extension:

- If the macroscopic evaluation does not show any suspicion of metastasis, a single section of a node should be avoided. The probability of detecting a metastasis on a central section is related to the size of the lymph node, the size of the lesion, and the location of the tumor within the node. ${ }^{49}$ To avoid this problem, it is recommended to obtain several sections of the nodes (2-mm slices in the longitudinal plane) and to examine each block separately. Thin sections of $2 \mathrm{~mm}$ may increase the workload of the pathologist but increase the detection rate of metastases. Small nodes can be sliced and embedded in one block if possible.

- There are different methods to detect additional metastatic deposits in lymph nodes like serial sectioning or immunohistochemistry (IHC). IHC using a cocktail of cytokeratins such as the anti-epithelial antibody mAb Ber-Ep4, AE1/AE3 is a sensitive and specific method for detecting isolated tumor cells or clusters of cells. Three levels of section are sufficient for this analysis. ${ }^{50}$ However, serial sectioning is relatively laborious and time-consuming and is, there- fore, not practical as routine practice..$^{51}$

The report from the pathologist should describe the number of lymph nodes removed and studied. The overall number of metastatic lymph nodes in each station should be clearly stated, as well as the status of the lymph node capsule.

\section{Conclusions}

Accurate staging remains essential for lung cancer management. Recent developments in both non-invasive and invasive staging investigations are changing the algorithms for lung cancer staging. For example, a negative PET scan for the mediastinum now means that mediastinoscopy is, in many cases, unnecessary while VATS offers a minimally invasive yet highly accurate means of pre-resection histological staging. The advances in techniques such as EBUS or EUS or autofluorescence bronchoscopy can safely provide solid evidence of the status of NSCLC before the patient is advised towards surgery. These imaging and surgical techniques will continue to evolve, and multidisciplinary (MDT) oncological teams should keep abreast of their developments. MDT meetings involve medical oncologists, clinical oncologists, pulmonologists, pathologists, radiologists and thoracic surgeons, all working together for the benefit of the patient with NSCLC with the most up-to-date techniques and guidelines available.

Intraoperative lymph node staging is mandatory. However, the technique of lymph node assessment during surgery for NSCLC has not yet been standardized. An accurate intra-operative staging is necessary to compare the results from different institutions and to conduct multiinstitutional trials. Systematic mediastinal lymph node dissection is recommended in all cases for complete resection of NSCLC, and improves pathological staging and the prospect for adjuvant therapy. The role of mediastinal lymphadenectomy in terms of overall survival and local control remains controversial, but systematic lymph node dissection might be associated with a better outcome in early stage NSCLC.

\section{References}

1. Jernal A, Thomas A, Murray T, Thun M. Cancer statistics, 2002. Cancer 2001;52:23-47.

2. Ihde DC, Minna JD. Non-small cell lung cancer: part I. Biology, diagnosis and staging. Curr Probl Cancer 1991;15:61-104.

3. Cerfolio RJ, Buddhiwardhan 0, Bryant AS, et al. The accuracy of integrated PET/CT compared with dedicated PET alone for the staging of patients with non-small cell lung cancer. Ann Thorac Surg 2004;78:1017-23.

4. Vansteenkiste J, Dooms C, De Leyn P. Early stage non-small-cell lung cancer: challenges in staging and adjuvant treatment: evidencebased staging. Ann Oncol 2010;21:189-95.

5. Goldstraw P, Ball D, Jett JR, et al. Non-small-cell lung cancer. Lancet 2011;378:1727-40.

6. Sobin L, Gospodarowicz M, Wittekind C, eds. TNM classification of malignant tumours. 7th ed. Oxford: Blackwell Publishing; 2010.

7. Goldstraw P, Crowley J; IASLC International Staging Project. The IASLC international staging project on lung cancer. J Thorac Oncol 2006;1:281-6.

8. Rami-Porta R, Ball D, Crowley JJ, et al. The IASLC Lung Cancer Staging Project: proposals for the revision of the T descriptors in the forthcoming (seventh) edition of the TNM classification for lung cancer. J Thorac Oncol 2007;2:593-602.

9. Rusch VR, Crowley JJ, Giroux DJ, et al. The IASLC Lung Cancer 
Staging Project: proposals for revision of the $\mathrm{N}$ descriptors in the forthcoming (seventh) edition of the TNM classification for lung cancer. J Thorac Oncol 2007;2:603-12.

10. Postmus PE, Brambilla E, Chansky K, et al. The IASLC Lung Cancer Staging Project: proposals for revision of the M descriptors in the forthcoming (seventh) edition of the TNM classifi cation for lung cancer. J Thorac Oncol 2007;2:686-93.

11. Goldstraw P, Crowley JJ, Chansky K, et al. The IASLC Lung Cancer Staging Project: proposals for revision of the stage groupings in the forthcoming (seventh) edition of the TNM classifi cation for lung cancer. J Thorac Oncol 2007;2:706-14.

12. Goldstraw P, ed. IASLC staging manual in thoracic oncology. 1st ed. Orange Park, FL: EditorialRx Press; 2009.

13. Silvestri GA, Littenberg B, Colice GL. The clinical evaluation for detecting metastatic lung cancer: a metaanalysis. Am J Resp Crit.Care Med 1995;152:225.

14. Toloza EM, Harpole L, McCrory DC. Noninvasive staging of non-small cell lung cancer: a review of the current evidence. Chest 2003;123:137S.

15. Grondin SC, Liptay MJ. Current concepts in the staging of non-small cell lung cancer. Surg Oncol 2002;11:181.

16. Sihoe AD, Yim AP. Lung cancer staging. J Surg Res 2004;117:92-106.

17. Strauss GM, Dominioni L. Lung cancer screening and the surgical oncologist: the controversy. Surg Oncol Clin North Am 1999;8:371.

18. Silvestri GA, Gould MK, Margolis ML, et al; American College of Chest Physicians. Noninvasive staging of non-small cell lung cancer: ACCP evidenced-based clinical practice guidelines. Chest 2007;132:178S-201S.

19. Dhillon SS, Dhillon JK, Yendamuri S. Mediastinal staging of nonsmall-cell lung cancer. Expert Rev Respir Med 2011;5:835-50.

20. Kerr KM, Lamb D, Wathen CG, et al. Pathological assessment of mediastinal lymph nodes in lung cancer: implications for non-invasive mediastinal staging. Thorax 1992;47:337-41.

21. McLoud TC, Bourgouin PM, Greenberg RW, et al. Bronchogenic carcinoma: analysis of staging in the mediastinum with CT by correlative lymph node mapping and sampling. Radiology 1992;182:319-23.

22. Hellwig D, Graeter TP, Ukena D, et al. 18F-FDG PET for mediastinal staging of lung cancer: which SUV threshold makes sense? J Nucl Med 2007;48:1761-6.

23. Lardinois D. Pre- and intra-operative mediastinal staging in nonsmall-cell lung cancer. Swiss Med Wkly 2011;141:w13168.

24. Gould MK, Kuschner WG, Rydzak CE, et al. Test performance of positron emission tomography and computed tomography for mediastinal staging in patients with non-small cell lung cancer: a metaanalysis. Ann Intern Med 2003;139:879-92.

25. van Tineteren H, Hekstra OS, Smit EF, et al. Effectiveness of positron emission tomography in the preoperative assessment of patients with suspected non-small cell lung cancer: the PLUS multicentre randomized trial. Lancet 2002;359:1388-93.

26. Lardinois D, Weder W, Hany TF, et al. Staging of non-small-cell lung cancer with integrated positron-emission tomography and computed tomography. N Engl J Med 2003;348:2500-7.

27. Maziak DE, Darling GE, Inculet RI, et al. Positron emission tomography in staging early lung cancer: a randomized trial. Ann Intern Med 2009;151:221-8.

28. Billé A, Pelosi E, Skanjeti A, et al. Preoperative intrathoracic lymph node staging in patients with non-small-cell lung cancer: accuracy of integrated positron emission tomography and computed tomography. Eur J Cardiothorac Surg 2009;36:440-5.

29. Kennedy TC, McWilliams A, Edell E, et al. Bronchial intraepithelial neoplasia/early central airways lung cancer: ACCP evidence-based clinical practice guidelines (2nd edition). Chest 2007;132:221S233S.

30. Piet AH, Lagerwaard FJ, Kunst PW, et al. Can mediastinal nodal mobility explain the low yield rates for transbronchial needle aspiration without real-time imaging? Chest 2007;131:1783-7.

31. Baram D, et al. Impact of rapid on-site cytologic evaluation during TBNA. Chest 2005;128:869-75.

32. Diacon A. Utility of rapid on-site evaluation of transbronchial needle aspirates. Respiration 2005;72:182-8

33. Rusch VW, Asamura H, Watanabe H, et al. The IASLC lung cancer staging project: a proposal for a new international lymph node map in the forthcoming seventh edition of the TNM classification for lung cancer. J Thorac Oncol 2009;4:679-83.

34. Herth FJ, Eberhardt R, Krasnik M, et al. Endobronchial ultrasoundguided transbronchial needle aspiration of lymph nodes in the radiologically and PET normal mediastinum in patients with lung cancer. Chest 2008;133:887-91.

35. Annema JT, Versteegh MI, Veselic M, et al. Endoscopic ultrasound added to mediastinoscopy for preoperative staging of patients with lung cancer. JAMA 2005;294:931-6.

36. De Leyn P, Lerut T. Conventional mediastinoscopy. MMCTS 2004;2005:1-10.

37. De Leyn P, Lardinois D, Van Schil P, et al. ESTS guidelines for preoperative lymph node staging for non-small cell lung cancer. Eur J Cardiothorac Surg 2007;32:1-8.

38. Detterbeck FC, Jantz M, Wallace M, et al. Invasive mediastinal staging of lung cancer: ACCP evidence-based clinical practice guidelines (2nd edition). Chest 2007;132:202S-220S.

39. Lardinois D, De Leyn P, Van Schil P, et al. ESTS guidelines for intraoperative lymph node staging in non-small cell lung cancer. Eur J Cardiothorac Surg 2006;30:787-92.

40. Goldstraw P. Report on the international workshop on intrathoracic staging. London. October 1996. Lung Cancer 1997;18:107-11.

41. Rami-Porta R, Wittekind C, Goldstraw P. Complete resection in lung cancer surgery: proposed definition. Lung Cancer 2005;49:25-33.

42. Naruke T, Tsuchiya R, Kondo H, et al. Lymph node sampling in lung cancer: how should it be done? Eur J Cardiothorac Surg 1999; $16: S 17-S 24$.

43. Ichinose Y, Kato H, Koike T, et al. Japanese Clinical Oncology Group. J Thorac Cardiovasc Surg 2001;122:803-8.

44. Verhagen A, Bootsma G, Tjan-Heijnen V, et al. FDG-PET in staging lung cancer. How does it change the algorithm? Lung Cancer 2004;44:175-81.

45. Fry WA. Assessment of operability and respectability in lung cancer. Malignant tumors of the lung. Berlin-Heidelberg: Springer; 2004. pp $179-82$.

46. Lardinois D, Suter H, Hakki H, et al. Morbidity, survival, and site of recurrence after mediastinal lymph-node dissection versus systematic sampling after complete resection for nonsmall cell lung cancer. Ann Thorac Surg 2005;80:268-74.

47. Keller SM, Adak S, Wagner H, Johnson DH. Mediastinal lymph node dissection improves survival in patients with stages II and IIIA NSCLC. Ann Thorac Surg 2000;70:358-66.

48. Silverberg SG, Connolly JL, Dabbs D, et al. Association of directors of anatomic and surgical pathology. Recommendations for processing and reporting of lymph node specimens submitted for evaluation of metastatic disease. Am J Clin Pathol 2001;115:799-801.

49. Wilkinson EJ, Hause L. Probability in lymph-node sectioning. Cancer 1974;33:1269-74.

50. Oosterhuis JW, Theunissen PH, Bollen EC. Improved pre-operative mediastinal staging in non-small cell lung cancer by serial sectioning and immunohistochemical staining of lymph-node biopsies. Eur J Cardiothorac Surg 2001;20:335-8.

51. Nicholson AG, Graham ANJ, Pezzella F, et al. Does the use of immunohistochemistry to identify micrometastases provide useful information in the staging of node-negative non-small cell lung carcinomas? Lung Cancer 1997;18:231-40. 